\title{
Inducing hibernation in the arctic ground squirrel
}

\begin{abstract}
Hibernation is essential for survival during seasonal deficiencies in food supply in several species of mammals. Torpor, or severe metabolic suppression, protects the body during the hibernating season by reducing resting metabolic rate and core body temperature. Seasonal hibernators, such as the arctic ground squirrel, follow a circannual cycle and are able to spontaneously enter torpor only during the hibernation season. A two-switch model of transition into the torpid state suggests that one physiological switch signals the onset of the hibernation season, and another switch initiates the onset of torpor.

The mechanism for transition into the torpid state, which includes altered central nervous system (CNS) control of thermoregulation, extended sleep, active inhibition of metabolism and temperaturedependent effects on metabolic rate, has been previously unknown. Now, a team led by Kelly L. Drew (University of Alaska, Fairbanks) has found that a seasonal change in the CNS response to a molecule called adenosine may provide that mechanism (J. Neurosci. 31, 10752-10758; 2011).
\end{abstract}

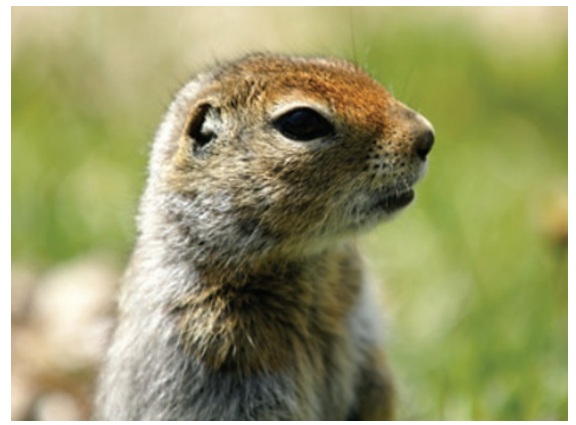

Signaling via A1 adenosine receptors (A1ARs), termed purinergic signaling, mediates sleep drive and decreases body temperature. In this study, blockade of the $\mathrm{A} 1 \mathrm{AR}$ receptors in the CNS using the antagonist CPT was shown to reverse torpor onset in all animals. This indicates that activation of A1AR is necessary for the onset of spontaneous torpor in the arctic ground squirrel.

Additionally, the A1AR agonist CHA induced a torpor-like effect in the animals that increased as the hibernation season progressed. These results indicate that A1AR activation within the CNS is necessary and sufficient to induce torpor during the hibernation season but not during the off-season when arctic ground squirrels do not spontaneously hibernate.

The results of this study show that the CNS regulates the onset of torpor via the activation of A1AR. In the context of the two-switch model, the seasonally increased sensitivity to central purinergic signaling serves as the first switch to signal the onset of hibernation; stimulation of central A1AR by endogenous adenosine serves as a second switch that induces torpor. The authors hypothesize that the mechanism underlying the first switch may involve changes in purinergic receptor expression or function; in extracellular levels of adenosine; or in neural circuits regulating sleep, metabolism or body temperature.

Understanding how hibernating mammals regulate metabolic suppression has potential to translate to improved therapies for conditions in which oxygen and energy supply fail to meet demand, including stroke, cardiac arrest, hemorrhagic shock and trauma.

\section{Kara Rosania}

\section{WHAT DOGS CAN TEACH US ABOUT BONES}

Osteosarcoma occurs spontaneously in humans (usually children) and in dogs with a disease course and severity that are difficult to predict. Some patients respond well to conventional therapies, have a less aggressive form of disease and may survive for decades without recurrence, whereas others respond poorly to treatment or experience recurrence and may survive less than 5 years. This variability has hindered accurate molecular classification of osteosarcomas.

Because spontaneous osteosarcoma occurs more frequently in certain breeds of dogs than in others, researchers hypothesized that it would be easier to identify molecular subtypes of osteosarcoma on the more genetically homogeneous canine background. They also believed that these molecular profiles could be used to similarly identify clinically relevant subtypes in human samples in order to better predict therapeutic success. The group, led by Jaime F. Modiano (University of Minnesota, Minneapolis), carried out extensive genetic analyses of tumor cell and whole tumor samples from dogs with osteosarcomas. They were able to group the tumors into two distinct branches (A and $B$ ) on the basis of differences in expression of two clusters of genes (Bone 49, 356-367; 2011).

Samples from independent data sets showed the same grouping and expression differences, indicating that the molecular signatures were robust across canine osteosarcomas. Next, the researchers assessed several sets of human osteosarcoma samples to determine whether the same molecular signatures were present. They observed clustering patterns similar to those identified in canines.

Having established that two subtypes of osteosarcoma could be identified in both dogs and humans, they next evaluated the clinical relevance of the two subtypes. Overall survival among dogs with branch A tumors was significantly lower than among those with branch B tumors. Time to metastasis was also shorter for dogs with branch A versus branch B tumors. In humans, branch B tumors were generally associated with better clinical outcome than branch A tumors.

"Our findings pave the way to develop laboratory tests that can predict the behavior of this tumor in dogs and children at the time of diagnosis," said Modiano in a press release. "This allows us to tailor individualized therapy to meet the patient's needs... patients with less aggressive disease could be treated conservatively, reducing the side effects and the risks associated with treatment, while patients with more aggressive disease could be treated with more intense therapy."

Monica Harrington 